IJCST

15,1

56

Received June 2002

Accepted October 2002

\section{Mechanical properties of fabric materials for draping simulation}

\author{
$\mathrm{Z}$. $\mathrm{Wu}$
}

Department of Mechanical Engineering, Hong Kong University

of Science and Technology, Kowloon, Hong Kong,

People's Republic of China

C.K. Au

School of Mechanical and Production Engineering,

Nanyang Technological University, Singapore

Matthew Yuen

Department of Mechanical Engineering, Hong Kong University

of Science and Technology, Kowloon, Hong Kong,

People's Republic of China

Keywords Fabrics, Drape, Mechanical properties

Abstract Most of the cloth simulation and modelling techniques rely on the energy function of the system. The geometric deformation is related to the energy function by the fabric material characteristics, which are usually difficult to measure directly. This paper discusses how the fabric material properties are related to the measurable mechanical properties of the fabric such as tensile modulus, Poisson's ratio etc. These properties are incorporated into a cloth simulator to produce draping results. The simulated image and real object are then compared to show the realism.

\section{Introduction}

Cloth modelling has received considerable attention recently. The applications of this modelling technique are mainly for graphic and engineering purpose. Both visual realism and physical accuracy are of equal interest in these two fields. A lot of researches about cloth modelling have been conducted, which mainly focuses on developing physically based models for the cloth object. These models can be classified into two categories: the continuum model and discrete model. For continuum model, the fabric is considered as continuous object with mass and energies distributed throughout. The governing equations are derived from the variational principle. For the discrete model, the object is modelled as a collection of point masses with some relations among each other. A cloth model can be continuous or discrete but the computational methods are ultimately discrete (Gibson and Miritich, 1997). In addition, these models always possess certain physical quantities, particularly the deformation energy, which is defined to afford simulation algorithms.
International Journal of Clothing Science and Technology Vol. 15 No. 1, 2003 pp. $56-68$ (C) MCB UP Limited 0955-6222 DOI 10.1108/09556220310461169 


\section{Literature review for cloth modelling}

The deformable surface proposed by Teropoulos and Fleischer (1988) is a typical continuum model for the cloth. Furthermore, various approaches (Chen et al., 1999; Teropoulos and Fleischer, 1988; Volino et al., 1995) are discussed to define the potential energy of a deformable model. The final equilibrium state of the model is obtained by minimizing the potential energy with respect to the material displacements. Finite element method (Eischen and Clapp, 1996; Tan et al., 1999) is also used to find an approximation for a continuous function that satisfies the equilibrium expression.

Particle system (Breen et al., 1992, 1994; Eberhardt et al., 1996) is a typical example of the discrete model for modelling of cloth. The cloth is represented by a set of particles. The energies of each particle are defined. The final equilibrium shape of the cloth occurs at the minimum energy of the whole particle system. Mass-spring system is another example of the discrete model for cloth object (Howelett, 1997; Provot, 1995). The cloth is modelled as a collection of point masses connected by springs in a lattice structure. This is a significant approximation of the true physics that occurs in the cloth. The current status in cloth modelling and animation research can be found in Volino and Magnenat-Thalmann (2000).

Recently, cloth modelling tends to focus on two major areas.

(1) Incorporating fabric property into the cloth model to give an accurate and realistic simulation (Breen et al., 1992, 1994; Eberhardt et al., 1996).

(2) Improving the computational efficiency to give a fast simulation (Baraff and Witkin, 1998).

Both are crucial in simulating the cloth since the demand of accuracy, realism and fast computation is increasing for engineering and graphic animation.

\section{Objective}

It can be seen that most of the cloth modelling techniques rely on the energy function of the system. The geometric deformation is related to the energy function by the fabric material characteristics, which are usually difficult to measure directly.

Kawabata Evaluation system (Kawabata, 1975) is a common equipment to measure the mechanical properties of a fabric. Breen et al. (1994) proposed a method to derive the energy equations of the cloth model from fabric measurement data produced by the Kawabata Evaluation System. Firstly, a function is determined to approximate the Kawabata plots. Then, the approximate function is related to the energy function of the model. Lastly, the resulting equations are scaled to produce energy values in standard physical units. Eischen and Bigliani (2000) used a fifth order polynomial to approximate the Kawabata plot and fitted the approximation into the constitutive equations to obtain the elastic constants of the fabric. 
IJCST

15,1

58
This paper discusses the characteristics of the fabric material in terms of a set of measurable mechanical properties. These properties are incorporated into a continuum model for draping simulation and the results are then compared with the actual situations.

\section{Cloth draping simulation}

Figure 1 shows the pseudo-codes of a cloth draping simulator based on Teropoulos and Fleischer (1988) continuum model. The state of a cloth element is described by its position $x$ and velocity $\dot{x}$. Based on this state, the cloth deformation is defined. For instance, the metric and curvature tensors are the typical deformation measurements. The behaviours of the cloth are characterized by the strain energy due to the deformation. Furthermore, internal forces are induced because of this strain energy. Applying the fundamental laws of dynamics such as Newton's second law of motion on these elements, the acceleration $\ddot{x}$ is obtained, which is used to calculate the new state of the element for the next time step through numerical integration. Once the new state is computed, collision detection is performed. Basically, there are two types of collision detection called cloth self-collision and cloth object collision. If the collision is detected at the new state, the new state will be given up position and velocity of cloth element at current instant flag to indicate the convergence of the state of the cloth element the deformation of cloth at current instant the strain energy due to deformation acceleration of the cloth element position and velocity of cloth element at next instant flag to indicate collision occurs function to detect the occurrence of collision function to compute the response of cloth element upon collision

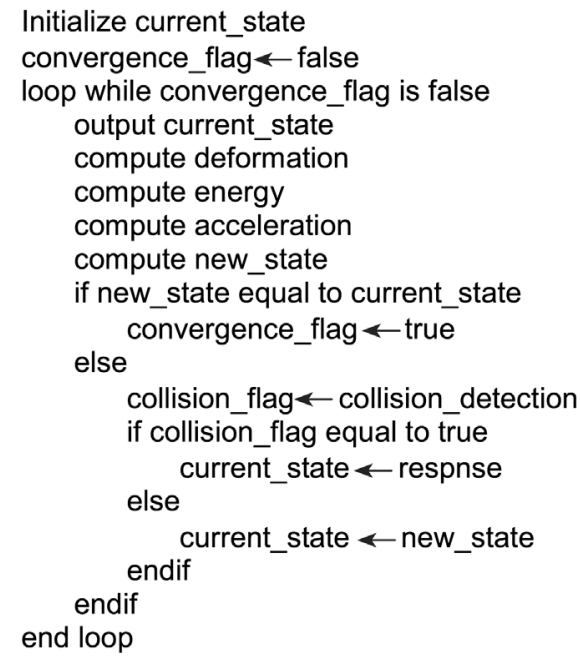

Figure 1.

The pseudo codes of a cloth draping simulator

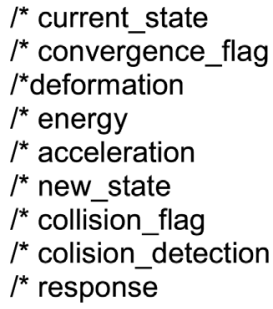

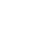


and collision responses are imposed as constraints at that instant. Both collision detection and response are implemented as separate module in the simulator for easy software management.

\section{Mechanical properties of fabric material}

The mechanical properties of a fabric material are measured by the Kawabata Evaluation System under the assumption that fabric is anisotropic and orthotropic in warp and weft directions. Three basic tests are performed: tensile, shear and bending test. Figure 2 shows the plotting of the tensile and bending test of a fabric sample with 60 per cent wool and 40 per cent polyester.

The mechanical behaviours of the cloth are governed by the strain energy accumulated due to deformation. Two main deformations occur in cloth draping: metric deformation due to in-plane forces and curvature deformation due to out-of-plane forces. The strain energy density $U$ is written as

$$
U=1 / 2 \sum \lambda^{\alpha \beta} C^{\alpha \beta \delta \gamma} \lambda_{\delta \gamma}
$$

where $\lambda_{\alpha \beta}$ is the strain tensor due to deformation and $C^{\alpha \beta \gamma \delta}$ is the elastic modulus tensors; $\alpha, \beta, \gamma$ and $\delta$ are indices to denote the directions of the principle axes; $\sum$ is the aggregation of the strain energies due to metric and curvature deformation.

The elastic modulus tensors $C^{\alpha \beta \delta \gamma}$ are symmetric tensors, hence

$$
C^{\alpha \beta \delta \gamma}=C^{\alpha \beta \gamma \delta}=C^{\beta \alpha \delta \gamma}=C^{\delta \gamma \alpha \beta}
$$

When the warp and weft directions coincide with the principle coordinate system of the fabric, $C^{1112}=C^{1211}=C^{1222}=C^{2212}=0$ and only five components of the tensor $C^{1111}, C^{1122}, C^{1212}, C^{2211}$ and $C^{2222}$, are non-zero.

From Hook's law,

$$
\begin{gathered}
{\left[\begin{array}{c}
\sigma_{11} \\
\sigma_{22} \\
\sigma_{12}
\end{array}\right]=\frac{1}{1-v_{1} \nu_{2}}\left[\begin{array}{ccc}
D_{1} & v_{2} D_{1} & 0 \\
v_{1} D_{2} & D_{2} & 0 \\
0 & 0 & D_{\mathrm{s}}\left(1-v_{1} \mathrm{v}_{2}\right)
\end{array}\right]\left[\begin{array}{c}
\varepsilon_{11} \\
\varepsilon_{22} \\
2 \varepsilon_{12}
\end{array}\right]} \\
{\left[\begin{array}{c}
\tau_{11} \\
\tau_{22} \\
\tau_{12}
\end{array}\right]=\frac{1}{1-\mu_{1} \mu_{2}}\left[\begin{array}{ccc}
H_{1} & \mu_{2} H_{1} & 0 \\
\mu_{1} H_{2} & H_{2} & 0 \\
0 & 0 & H_{\mathrm{s}}\left(1-\mu_{1} \mu_{2}\right)
\end{array}\right]\left[\begin{array}{c}
\kappa_{11} \\
\kappa_{22} \\
2 \kappa_{12}
\end{array}\right]}
\end{gathered}
$$

where $\sigma$ and $\tau$ are the stress due to metric and curvature deformation, respectively; $\varepsilon$ and $\kappa$ are the strain due to metric and curvature deformation, respectively; $D_{1}, D_{2}$ are the tensile modulus in the principal axes; $D_{\mathrm{s}}$ are the shear modulus; $v_{1}, v_{2}$ are Poisson's ratio; $H_{1}, H_{2}$ are the bending modulus in
Mechanical properties of fabric materials 


\section{IJCST}

15,1

\section{0}

Figure 2.

Tensile and bending test result from the Kawabata Evaluation System
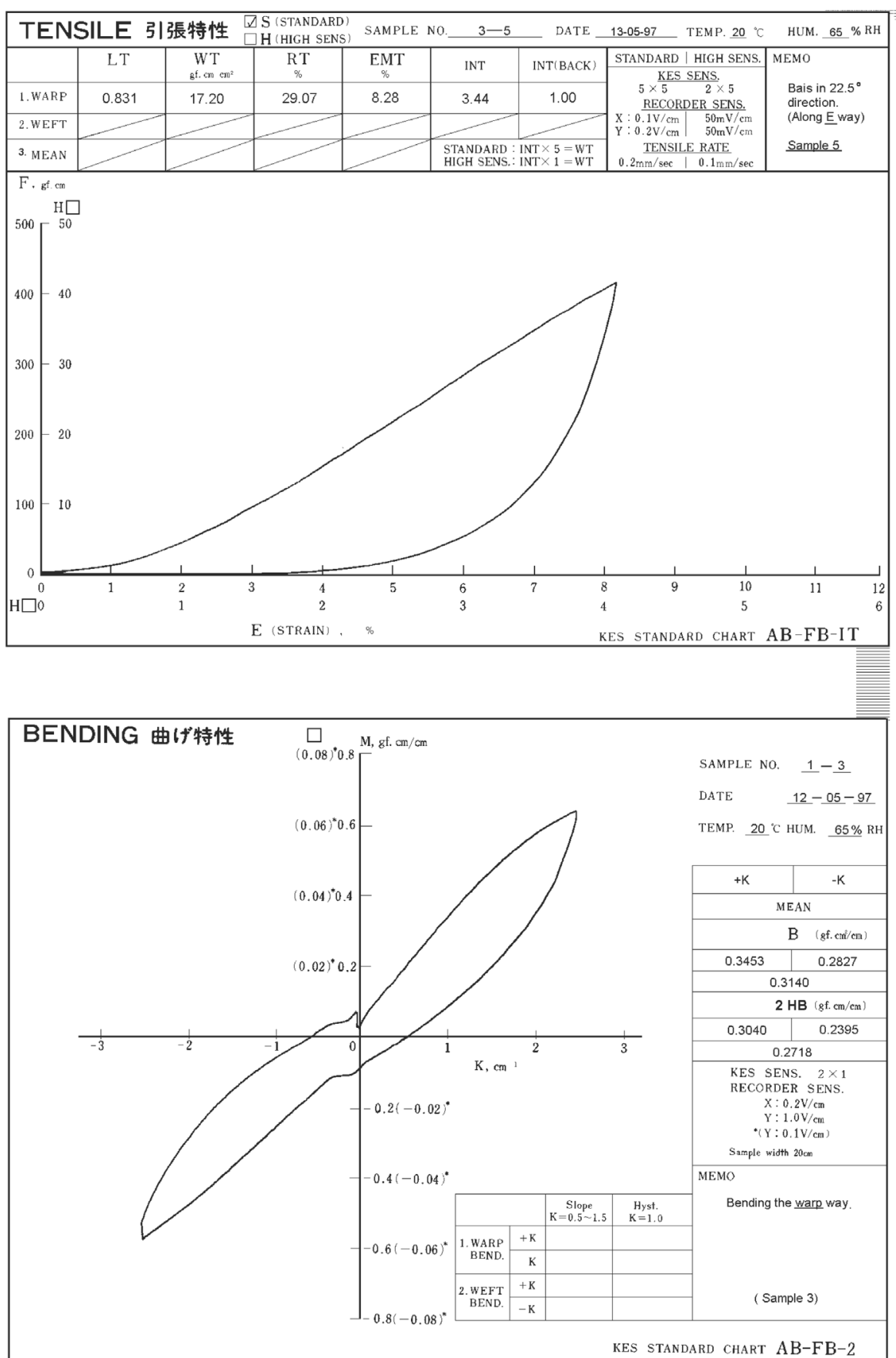
the principal axes; $H_{\mathrm{s}}$ are the twisting modulus; $\mu_{1}, \mu_{2}$ are physical quantities analogue to the Poisson's ratios.

$$
v_{1} D_{2}=v_{2} D_{1}
$$

and

$$
\mu_{1} H_{2}=\mu_{2} H_{1}
$$

Hence, the material modulus tensors can be expressed in terms of a set of measurable quantities:

$$
\begin{aligned}
& C_{G}^{1111}=\quad \frac{D_{1}}{1-v_{1} v_{2}} \\
& C_{G}^{1122}=C_{G}^{2211}=\frac{\mathrm{v}_{2} D_{1}}{1-\mathrm{v}_{1} \mathrm{v}_{2}} \\
& C_{G}^{2222}=\quad \frac{D_{2}}{1-v_{1} v_{2}} \\
& C_{G}^{1212}=\quad D_{\mathrm{s}} \\
& C_{B}^{1111}=\quad \frac{H_{1}}{1-\mu_{1} \mu_{2}} \\
& C_{B}^{1122}=C_{B}^{2211}=\frac{\mu_{2} H_{1}}{1-\mu_{1} \mu_{2}} \\
& C_{B}^{2222}=\quad \frac{H_{2}}{1-\mu_{1} \mu_{2}} \\
& C_{B}^{1212}=\quad H_{\mathrm{s}}
\end{aligned}
$$

where the $C_{G}$ and $C_{B}$ are the elastic modulus tensors due to metric and curvature deformation respectively.

If the cloth is stretched in a direction making an angle $\theta$ with one of the principal axis direction, the tensile strain $\epsilon_{\theta}$ is related to the tensile stress $\sigma_{\theta}$ as

$$
D_{\theta}=\frac{\sigma_{\theta}}{\varepsilon_{\theta}}
$$

Similarly, the bending strain $\kappa_{\theta}$ and the bending moment $\tau_{\theta}$ can be expressed as

$$
H_{\theta}=\frac{\tau_{\theta}}{\kappa_{\theta}}
$$

According to the transformation laws of tensor,

$$
\varepsilon_{\theta}=\left[\begin{array}{lll}
\cos ^{2} \theta & \sin ^{2} \theta & \sin \theta \cos \theta
\end{array}\right]\left[\begin{array}{c}
\varepsilon_{11} \\
\varepsilon_{22} \\
2 \varepsilon_{12}
\end{array}\right]
$$


IJCST

15,1

62

$$
\left[\begin{array}{c}
\sigma^{11} \\
\sigma^{22} \\
\sigma^{12}
\end{array}\right]=\left[\begin{array}{c}
\sigma^{\theta} \cos ^{2} \theta \\
\sigma^{\theta} \sin ^{2} \theta \\
-\sigma^{\theta} \cos \theta \sin \theta
\end{array}\right]
$$

and

$$
\begin{gathered}
\kappa_{\theta}=\left[\begin{array}{lll}
\cos ^{2} \theta & \sin ^{2} \theta & \sin \theta \cos \theta
\end{array}\right]\left[\begin{array}{c}
\kappa_{11} \\
\kappa_{22} \\
2 \kappa_{12}
\end{array}\right] \\
{\left[\begin{array}{c}
\tau^{11} \\
\tau^{22} \\
\tau^{12}
\end{array}\right]=\left[\begin{array}{c}
\tau^{\theta} \cos ^{2} \theta \\
\tau^{\theta} \sin ^{2} \theta \\
-\tau^{\theta} \cos \theta \sin \theta
\end{array}\right]}
\end{gathered}
$$

Derivation of equations (9)-(14) can be found in most common strength of materials literatures (Ryder, 1973).

Substituting equations (11)-(14) into equations (9) and (10) and combining with equations (3) and (4) yields the tensile modulus and the bending modulus in this direction

$$
\begin{aligned}
& \frac{1}{D_{\theta}}=\frac{1}{D_{1}} \cos ^{4} \theta+\left(\frac{1}{D_{\mathrm{s}}}-\frac{v_{1}}{D_{1}}-\frac{v_{2}}{D_{2}}\right) \cos ^{2} \theta \sin ^{2} \theta+\frac{1}{D_{2}} \sin ^{4} \theta \\
& \frac{1}{H_{\theta}}=\frac{1}{H_{1}} \cos ^{4} \theta+\left(\frac{1}{H_{\mathrm{s}}}-\frac{\mu_{1}}{H_{1}}-\frac{\mu_{2}}{H_{2}}\right) \cos ^{2} \theta \sin ^{2} \theta+\frac{1}{H_{2}} \sin ^{4} \theta
\end{aligned}
$$

Since the "Poisson ratio" of bending $\mu_{1}$ and $\mu_{2}$ are usually small, they can be neglected. Taking the angle $\theta=45^{\circ}$, the tensile Poisson ratio and the twisting modulus are written as

$$
v_{1}=\frac{1}{2} D_{1}\left(\frac{1}{D_{1}}+\frac{1}{D_{2}}+\frac{1}{D_{\mathrm{s}}}-\frac{4}{D_{45^{\circ}}}\right), \quad v_{2}=D_{2} \frac{v_{1}}{D_{1}}
$$




$$
H_{\mathrm{S}}=\left(\frac{4}{H_{45^{\circ}}}-\frac{1}{H_{1}}-\frac{1}{H_{2}}\right)^{-1}
$$

Hence the mechanical properties of modulus $D_{1}, D_{2}, D_{\mathrm{s}}, H_{1}, H_{2}$ and $H_{\mathrm{s}}$ can be measured directly by the Kawabata Evaluation System. Other parameters such as Poisson's ratio and twisting modulus are obtained from the tensile, shear and bending modulus by using equations (17) and (18).

\section{Simulation examples}

A set of examples is used to illustrate the effects on cloth draping simulation with the incorporation of the mechanical properties of the fabrics.

Example 1. A piece of cloth falling on a sphere.

The mechanical properties of the fabric are listed in Table I. The size of the cloth is $1 \times 1 \mathrm{~m}$ with meshing size of $50 \times 50$ nodes and the radius of the sphere is $0.125 \mathrm{~m}$. The animation process is shown in Figure 3 .

Example 2. Cloth draping over a sphere with various fabric materials.

The bending modulus of the cloth is changed to show the draping effects. Three cases are performed:

Case 1 , the bending modulus is enlarged by 5 times.

Case 2, the original bending modulus is used.

Case 3 , the bending modulus is reduced to $1 / 5$ of the original values.

The draping behaviour is shown in Figure 4. The more rigid fabric in case 1 gives a 4 -wrinkle draping mode while the softer fabric generates an 8-wrinkle draping mode.

Example 3. Cloth draping over a sphere with various fabric materials.

Two pieces of cloth with different fabric materials draping over a sphere is shown in Figure 5. The results are compared with the real images. The size of the cloth is $0.6 \times 0.6 \mathrm{~m}$ and the radius of the sphere is $0.09 \mathrm{~m}$. The properties are listed in Table II.

Example 4. Table cloth draping with various fabric materials.

The same simulation in example 3 is performed by using a cloth with varying fabric materials. The mechanical properties are listed in Table III.

Each simulation is then compared with the appearance of real table cloth situations as shown in Figure 6.

Example 5. Dresses with various fabric materials.

Three typical fabric materials, cotton, polyester and silk, are listed in Table IV. The results are simulated with a mannequin as shown in Figure 7.

\begin{tabular}{|c|c|c|c|c|c|c|c|c|}
\hline$\rho \mathrm{kg} / \mathrm{m} 2$ & $D_{1} \mathrm{~N} / \mathrm{m}$ & $D_{2} \mathrm{~N} / \mathrm{m}$ & $D_{\mathrm{s}} \mathrm{N} / \mathrm{m}$ & $H_{1} \mu \mathrm{Nm}$ & $H_{2} \mu \mathrm{Nm}$ & $H_{\mathrm{s}} \mu \mathrm{Nm}$ & $v_{1}, v_{2}$ & Table I. \\
\hline 0.125 & 3,657 & 3,627 & 36.8 & 5.07 & 4.41 & 1.55 & $0.096,0.086$ & of cotton and ray \\
\hline
\end{tabular}




\section{IJCST \\ 15,1}

64

\section{Figure 3.}

The animation of cloth falling on a sphere

Figure 4.

Draping behaviours with various fabric materials

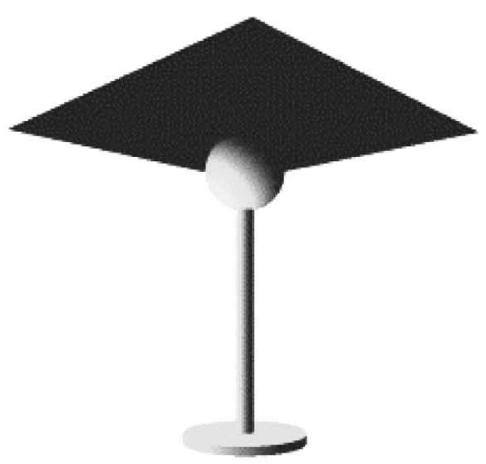

(1)

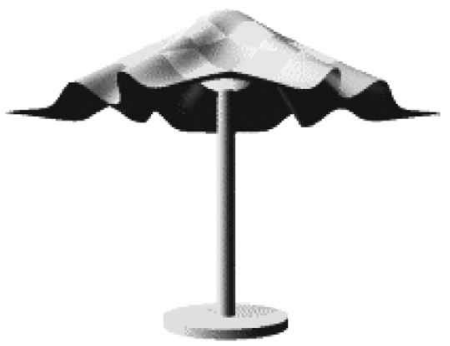

(3)

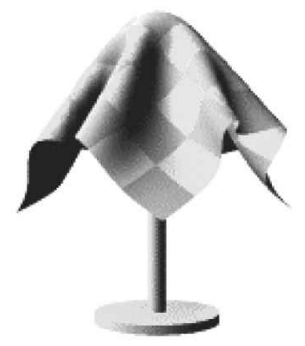

(5)

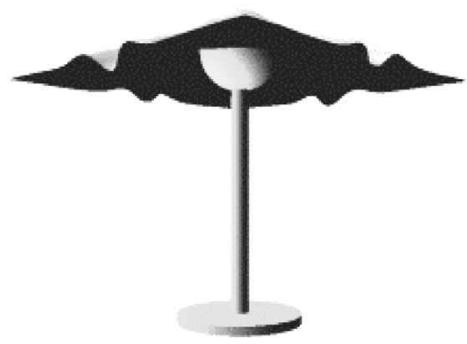

(2)

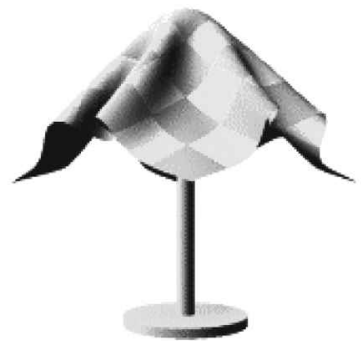

(4)

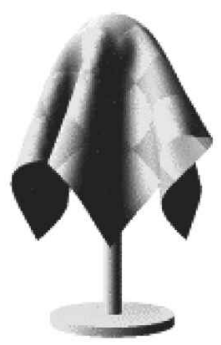

(6)

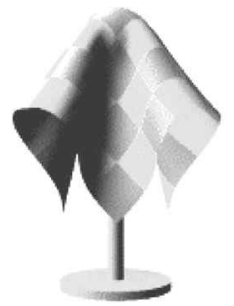

Case 1

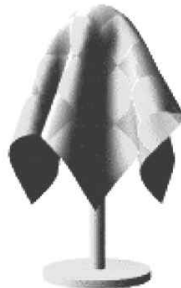

Case 2

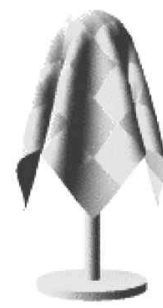

Case 3 


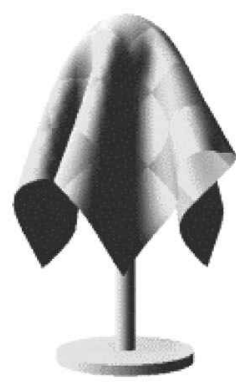

Simulated image of cotton sample 1

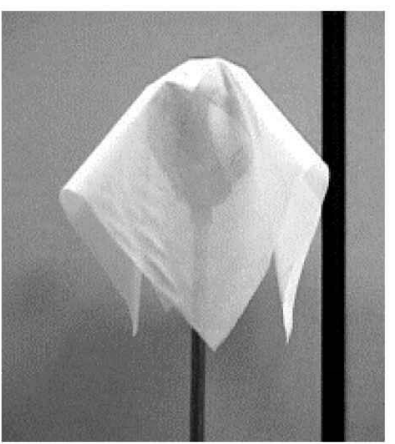

Real image of cotton sample 2

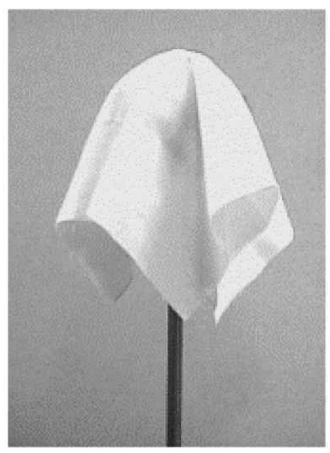

Real image of cotton sample 1

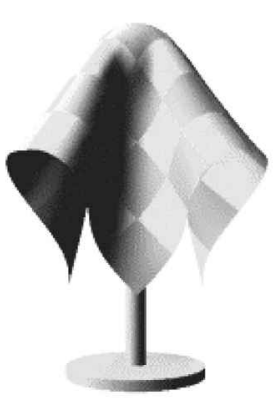

Simulated image of cotton sample 2

\section{Mechanical \\ properties of fabric materials}

65
Figure 5.

Different types of cotton cloth draping over a sphere
Material

sample

Cotton

Cotton 2

$\rho \mathrm{kg} / \mathrm{m}^{2}$

$D_{1} \mathrm{~N} / \mathrm{m}$

$D_{2} \mathrm{~N} / \mathrm{m}$

$D_{\mathrm{s}} \mathrm{N} / \mathrm{m}$

$H_{1} \mu \mathrm{Nm}$

87.7

87.7

11.6

$\begin{array}{lll}2,405 & 5,315 \quad 39.6\end{array}$

$2,405 \quad 5,315 \quad 39.6$

11.6

\begin{abstract}
(

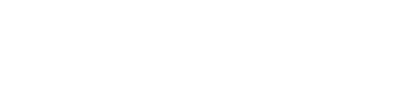

\title{
Material
}

sample

Cotton

$\rho \mathrm{kg} / \mathrm{m}^{2}$

$D_{1} \mathrm{~N} / \mathrm{m}$

0.208

$\frac{D_{1} \mathrm{~N} / \mathrm{m}}{3,475}$

$D_{2} \mathrm{~N} / \mathrm{m}$

Rayon

0.208

$3,475 \quad 2,865$

0.129

1,847

3,644

\begin{tabular}{ccccc}
$D_{\mathrm{s}} \mathrm{N} / \mathrm{m}$ & $H_{1} \mu \mathrm{Nm}$ & $H_{2} \mu \mathrm{Nm}$ & $H_{\mathrm{s}} \mu \mathrm{Nm}$ & $v_{1}, v_{2}$ \\
\hline 191 & 17.6 & 12.7 & 2.7 & 0.215 \\
& & & & 0.177 \\
15.2 & 4.4 & 2.3 & 0.71 & 0.185 \\
& & & & 0.165
\end{tabular}

\begin{abstract}
2
\end{abstract}




\section{IJCST}

15,1

\section{6}

Figure 6.

Table cloth simulation with different fabric materials

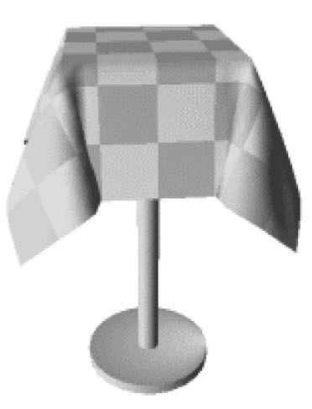

simulated result
(a) Cotton

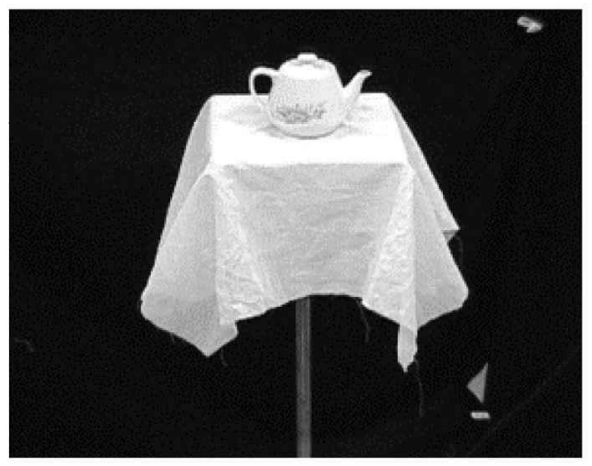

real image

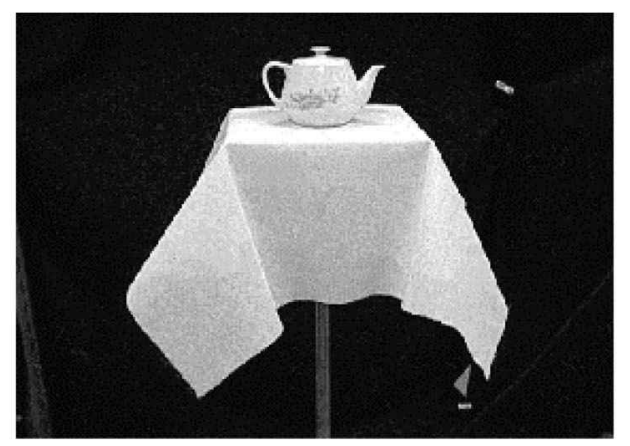

real image

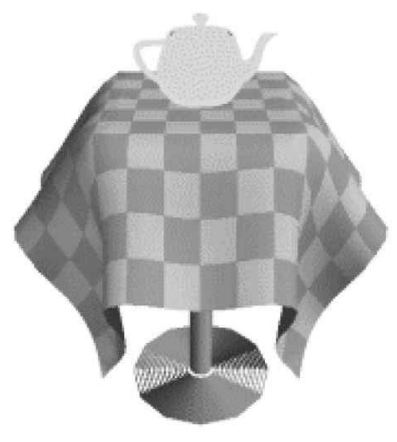

simulated result

(b) Rayon

\section{Table IV.}

Material properties of cotton, polyester and silk

\begin{tabular}{lrrrrrrrr}
\hline $\begin{array}{l}\text { Material } \\
\text { sample }\end{array}$ & $\rho \mathrm{kg} / \mathrm{m}^{2}$ & $D_{1} \mathrm{~N} / \mathrm{m}$ & $D_{2} \mathrm{~N} / \mathrm{m}$ & $D_{\mathrm{s}} \mathrm{N} / \mathrm{m}$ & $H_{1} \mu \mathrm{Nm}$ & $H_{2} \mu \mathrm{Nm}$ & $H_{\mathrm{s}} \mu \mathrm{Nm}$ & $v_{1}, v_{2}$ \\
\hline Cotton & 0.208 & 3,475 & 2,865 & 191 & 17.6 & 12.7 & 2.7 & $0.215,0.177$ \\
Polyester & 0.212 & 3,071 & 1,823 & 46.7 & 10.9 & 9.0 & 2.31 & $0.264,0.169$ \\
Silk & 0.076 & 1,388 & 827 & 12.4 & 0.81 & 0.76 & 0.089 & $0.285,0.170$ \\
\hline
\end{tabular}

\section{Discussion}

Fabric is made from threads in woven or knitted patterns with the structures resulting from different weaving or knitting techniques. Due to the complexity of the fabric microstructure, it is necessary and practical to treat the fabric as an engineering material in draping modelling. One of the feasible solutions is to 


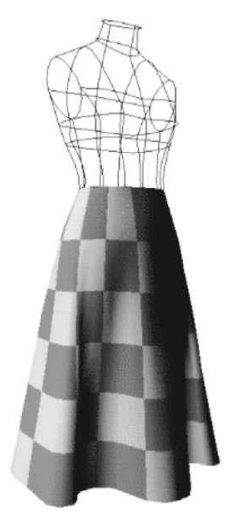

Cotton dress

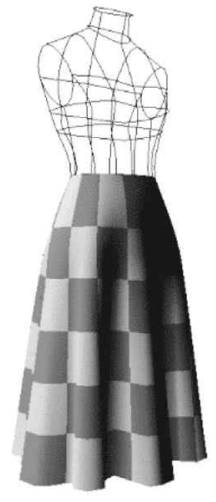

Polyester dress

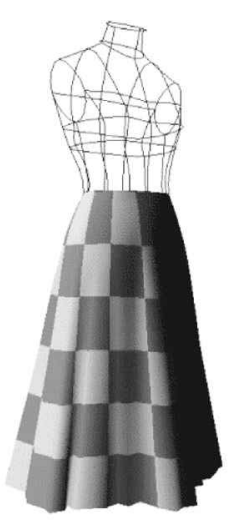

Silk dress

\section{Mechanical properties of fabric materials}

67

Figure 7.

Dresses simulation with various fabric materials

assume the fabric to be continuous elastic in both the modelling and the experimental aspects. Hence, it is equivalent to modelling the draping of a fabric sheet. Fabric material exhibits the orthogonal anisotropy behaviours, which leads to different mechanical properties in the weft and warp direction. Figure 8 shows the tensile modulus of cloth. The mechanical properties of the fabric are considered in three different directions: the weft and warp directions; and an angle of $45^{\circ}$ from one of the weft direction. Once these properties are measured and calculated, they can be inserted into the cloth model for draping simulation.

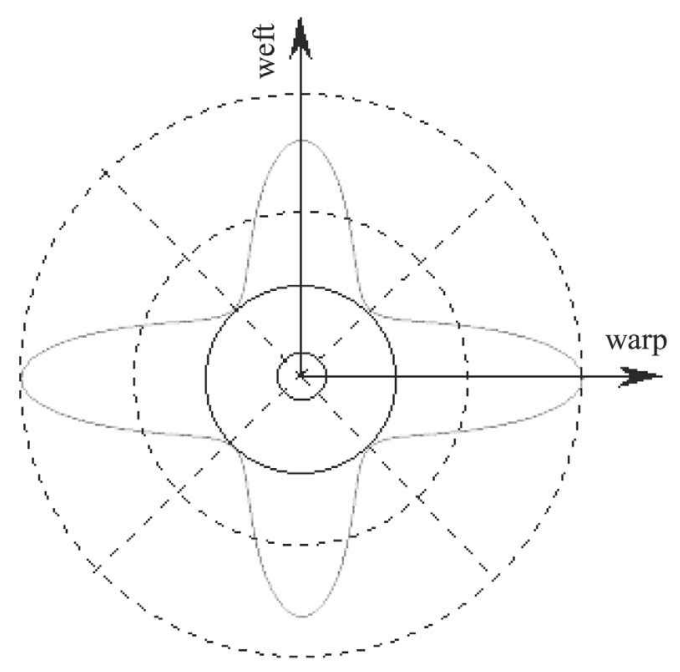

Figure 8.

The tensile modulus of cloth 
IJCST

15,1

68

\section{References}

Baraff, D. and Witkin, D. (1998), "Large steps in cloth simulation”, SIGGRAPH'98, Computer Graphics Proceedings, Annual Conference Series, pp. 43-54.

Breen, D.E., House, D.H. and Getto, P.H. (1992), "A physically-based particle model of woven cloth”, Visual Computer, Vol. 8 No. 5-6, pp. 264-77.

Breen, D.E., House, D.H. and Wozny, M.J. (1994), "Predicting the drape of woven cloth using interacting particles", Computer Graphics (SIGGRAPH'94 Proceedings), Addison-Wesley, Reading, MA, pp. 365-72.

Chen, M.X., Wu, Z., Sun, Q.P. and Yuen, M.M.F. (2003), “A wrinkled membrane model for cloth draping with multigrid acceleration”, Journal of Manufacturing Science and Engineering (in press).

Eberhardt, B., Weber, A. and Strasser, W. (1996), "A fast, flexible, particle-system model for cloth draping”, IEEE Computer Graphics and Applications, pp. 52-9.

Eischen, J.W. and Bigliani, R. (2000), “Continuum versus particle representation”, in House, D.H. and Breen, D.E. (Eds), Cloth Modeling and Animation, A.K. Peters, pp. 79-122.

Eischen, J.W. and Clapp, T.G. (1996), "Finite-element modelling and control of flexible fabric parts", IEEE Computer Graphics and Applications, pp. 71-80.

Gibson, S.F.F. and Miritich, B. (1997), "A survey of deformable modeling in computer graphics”, Technical report TR-97-19, Mitsubish Electric Research Laboratory.

Howelett, P. (1997), "Cloth simulation using mass-spring networks", MSc dissertation, Department of Computer Science, University of Manchester.

Kawabata, S. (1975), "The standardization and analysis of hand evaluation”, Hand Evaluation and Standardization Committee of the Textile Machinery Society of Japan, Osaka.

Provot, X. (1995), "Deformation constraints in a mass-spring model to describe rigid cloth behavior”, Proceedings of Graphics Interface, pp. 147-54.

Ryder, G.H. (1973), Strength of Materials, ELBS and Macmillan, NY.

Tan, S.T., Wong, T.N., Zhao, Y.F. and Chen, W.J. (1999), “A constrained finite element method for modelling cloth deformation”, Visual Computer, Vol. 15 No. 2, pp. 90-9.

Teropoulos, D. and Fleischer, K. (1988), "Deformable models", Visual Computer, Vol. 4 No. 6, pp. 306-31.

Volino, P. and Magnenat-Thalmann, N. (2000), Virtual Clothing Theory and Practice, Springer, Berlin.

Volino, P., Courchesne, M. and Thalmann N.M. (1995), "Versatile and efficient techniques for simulating cloth and other deformable objects", SIGGRAPH'95, Computer Graphics Proceedings, Annual Conference Series, pp. 137-44. 